Educación Física y Ciencia, vol. 20, nº 1, e043, 2018. ISSN 2314-2561

Universidad Nacional de La Plata.

Facultad de Humanidades y Ciencias de la Educación.

Departamento de Educación Física

\title{
Preparación del personal de los centros deportivos ante un ataque cardiaco súbito
}

Gavotto-Nogales, Omar Iván; Flores-Moreno, Pedro Julián; Bernal-Reyes, Fernando; Romero-Pérez, Ena Monserrat; Gavotto-Nogales, Hugo; Toledo-Domínguez, Iván

Cita sugerida: Gavotto-Nogales, O. I. ; Flores-Moreno, P. J. ; Bernal-Reyes, F.; Romero-Pérez, E. M.;

Gavotto Nogales, H. y Toledo-Domínguez, I. (2018). Preparación del personal de los centros

deportivos ante un ataque cardiaco súbito. Educación Física y Ciencia, 20(1), e043.

https://doi.org/10.24215/23142561e043 


\section{Preparación del personal de los centros deportivos ante un ataque cardiaco súbito}

Preparation of sports centers staff in case of sudden cardiac arrest

Omar Iván Gavotto-Nogales

Universidad de Sonora, México

ogavotto@gmail.com

Pedro Julián Flores-Moreno

Universidad de Colima, México

pedrojulian_flores@ucol.mx

Fernando Bernal-Reyes

Universidad de Sonora, México

fernando.bernal@unison.mx

Ena Monserrat Romero-Pérez

Universidad de Sonora, México

ena.romero@unison.mx

Hugo Gavotto-Nogales

Universidad Estatal de Sonora, México

hugogn@hotmail.com

Iván Toledo-Dominguez

Instituto Tecnológico de Sonora, México

ivan.toledo@itson.edu.mx

\section{ReSUMEN:}

La investigación tiene como objetivo identificar la preparación del personal que labora en los gimnasios y centros deportivos para proporcionar reanimación cardiopulmonar ante un ataque cardiaco súbito. Se obtuvo una muestra aleatoria para aplicar una encuesta a 12 centros deportivos en Hermosillo, Sonora, México. El 58,33 \% de los responsables de los centros deportivos confirmaron que cuentan con personal capacitado en reanimación cardiopulmonar, pero el $100 \%$ del personal desconocen el uso del desfibrilador. Se confirmó que ningún centro deportivo cuenta con desfibrilador para brindar auxilio en caso de paro cardiaco súbito. En el análisis de los planes de estudio de seis carreras relacionadas con la actividad física y deporte, presenta una asignatura optativa sobre primeros auxilios, que incluye como contenido básico la reanimación cardiopulmonar y sólo dos de cada 100 estudiantes de carreras relacionadas con la actividad física y deporte, confirman haber recibido capacitación en la reanimación cardiopulmonar. Se sugiere que el personal que labora en los centros deportivos y gimnasios reciba capacitación sobre las maniobras de reanimación cardiopulmonar y el uso del desfibrilador. Se propone que el $100 \%$ del personal adquiera las destrezas para ejecutar las maniobras de RCP, con base en los parámetros y procedimientos internacionales.

Palabras Clave: Primeros auxilios, Ataque cardiaco, Deporte, Reanimación, Preparación.

\section{Abstract:}

The research aims to identify the preparation of personnel working in gyms and sports centers to provide first aid in case of sudden cardiac arrest. A random sample was obtained to implement a survey to 12 sports centers in Hermosillo, Sonora, Mexico. 58,33\% of those responsible for sports centers have confirmed that their staff is CPR trained, but $100 \%$ of the staff do not know how to use a defibrillator. It was confirmed that no sports center has defibrillator to provide assistance in case of sudden cardiac arrest. In the analysis of curricula, six careers related to physical activity and sport have an elective course on first aids, which includes as basic content CPR and only two out of every 100 students related to physical activity and sport careers, confirmed having received training in CPR. It is suggested that the staff working in the sport and fitness centers receive training on cardiopulmonary resuscitation and defibrillator use. It is proposed that $100 \%$ of staff acquire the skills to perform CPR, based on international parameters and procedures. 
KEYWORDS: First aid, Heart attack, Sport, Resuscitation, Training.

\section{INTRODUCCIÓN}

La falta de actividad física, coloca a las personas en situación vulnerable, desencadenando un factor de riesgo para múltiples enfermedades como las cardiovasculares, diabetes, sobrepeso, obesidad y algunos tipos de cáncer (Hoyos, Castro, Hoyos, Chávez y Plasencia, 2011). Sin embargo, realizar ejercicio físico intenso cuando se padece una enfermedad cardiaca, puede ser causa de muerte.

"La incidencia del deporte sobre el corazón es motivo de interés por parte de la comunidad científica. El estudio de estos ítems está enfocado desde múltiples perspectivas y su producción se centra en biomedicina y ciencias de la salud" (Martínez-Morilla, Ruiz-Caballero, Brito-Ojeda y Navarro-Valdivielso, 2012, p. 300).

En los últimos años se ha incrementado el fenómeno de muerte súbita en el mundo, como consecuencia de enfermedades cardiacas no diagnosticadas. Ante esta situación, se requiere de una exploración física meticulosa para alertar sobre la presencia de cardiopatías, pero desgraciadamente la mayoría de los deportistas con patologías de riesgo son asintomáticos, tienen una exploración normal y presentan un excelente rendimiento físico. La inclusión del electrocardiograma de reposo aumenta la probabilidad de identificar a los sujetos en riesgo, ya que resulta anormal en el $95 \%$ de los casos de miocardiopatía hipertrófica y está alterado en la miocardiopatía arritmogénica y los síndromes del QT largo y de Wolff-Parkinson-White. Sin embargo, el electrocardiograma no tiene capacidad para detectar las anomalías de las arterias coronarias, por lo que "se ha propuesto la inclusión de la ecocardiografía en los protocolos de cribado de enfermedades cardiovasculares para grandes grupos de población o al menos en deportistas que van a realizar actividad deportiva programada" (Boraita, 2002, p.31).

Aunque la incidencia de la muerte súbita en el deporte es relativamente baja, cifrándose a nivel internacional entre 0,16 y 3,76 por 100.000 habitantes/año, y cuando se produce está especialmente relacionada con patología cardiovascular (Arimany-Manso y Bayés de Luna, 2011). Sin embargo, se debe considerar que la enfermedad coronaria es la primera causa de muerte en el mundo y su manifestación más alta es la muerte súbita (MS), la cual representa más de $60 \%$ de estas muertes (Rojas, Aizman, Arab, Utili y Andresen, 2012). Resulta impactante cuando la muerte súbita se presenta en deportistas bien entrenados o bien en individuos jóvenes aparentemente sanos.

En contraposición a todos los beneficios que conlleva realizar ejercicio físico, el deporte competitivo incrementa sensiblemente el riesgo de sufrir una muerte súbita durante la realización de una actividad física intensa. Las personas que practican actividad deportiva intensa presentan una incidencia mayor de muerte súbita que las no deportistas, 1,6 muertes por 100.000 frente a 0,75 por 100.000 . Además, la edad condiciona la muerte súbita durante la actividad deportiva, siendo en los menores de 35 años el riesgo menor, mientras que en los mayores de 35 años el riesgo de sufrir una muerte súbita se incrementa. Sin embargo, la prevención de la muerte súbita asociada con el deporte puede disminuir a través del reconocimiento cardiológico preparticipación deportiva (RCPD) y la instauración de los mecanismos necesarios para una resucitación cardiopulmonar y desfibrilación sin demora (Boraita, 2002).

Los primeros auxilios que debe recibir una persona con paro cardiaco es la maniobra denominada reanimación cardiopulmonar $(\mathrm{RCP})$ la cual representa la asistencia inmediata y temporal por parte de cualquier persona que se encuentre cerca de la víctima en ese momento (Martín-Recio, 2009).

Por lo que resulta de vital importancia que los equipos socorristas, médicos y los profesionales de la salud tengan los conocimientos y destrezas en RCP para salvar la vida de personas que sufren un ataque cardiaco súbito. Sin embargo, en las evaluaciones de los conocimientos y destrezas en RCP de 48 médicos egresados de distintas universidades chilenas, demostraron resultados regulares y bajos en la mayor parte de los elementos y etapas para proporcionar la RCP, obteniendo un promedio de 49,25, en escala de 0 a 100 (Rojas, Aizman, 
Arab, Utili y Andresen, 2012). La mayoría de los médicos evaluados reconoce a su vez que su formación médica de pregrado fue insuficiente en RCP y el uso del desfibrilador automático externo.

A continuación, se presentan los resultados de la evaluación práctica de los médicos participantes (Ver Tabla 1).

\begin{tabular}{|c|c|}
\hline Destrezas evaluadas & $\begin{array}{c}\% \text { de los } \\
\text { resultados }\end{array}$ \\
\hline Verifica inconsciencia & 98 \\
\hline Solicita ayuda & 99 \\
\hline Abre adecuadamente la via área & 56 \\
\hline Verifica presencia de respiración & 76 \\
\hline Verifica presencia de pulso carotideo & 19 \\
\hline Realiza 2 ciclos de 30 compresiones: 2 ventilaciones & 31 \\
\hline Realiza compresiones torácicas con frecuencia 100 a & 14 \\
\hline Realiza ventilaciones efectivas & \\
\hline Solicita el desfibrilador antes de los 30 segundos & 41 \\
\hline Selecciona la energia de descarga correcta & 56 \\
\hline Realiza descarga eléctrica antes de 30 segundos & 31 \\
\hline Sigue la secuencia ABCD adecuadamente & 12 \\
\hline
\end{tabular}

Tabla 1. Destrezas evaluadas y resultados en porcentajes aprobatorios. Rojas, Aizman, Arab, Utili y Andresen (2012)

Otro estudio realizado en Brasil a 91 enfermeros en la Región Metropolitana de Campinas sobre el conocimiento que tenían sobre paro cardiaco y reanimación cardiopulmonar se registró un promedio de 5,2 en escala de 0 a 10, obteniendo una calificación reprobatoria (Almeida, Araújo, Dalri y Araujo, 2011). Lamentablemente se confirma la existencia de una inadecuada e insipiente preparación del personal de salud en las maniobras de reanimación cardiopulmonar, situación que seguramente se puede presentar en otros países latinoamericanos, por lo que denota una necesidad su estudio, para el emprendimiento de acciones que puedan compensar esta situación.

Resulta incierto el desempeño de los profesionales médicos y de enfermería para proporcionar la RCP, a pesar de que estos profesionales se han formado específicamente para actuar ante situaciones de urgencia cuando un ser humano está en riesgo de muerte; la correcta ejecución de las maniobras de reanimación cardiopulmonar y su prontitud resultan determinantes para salvar la vida o evitar un daño cerebral permanente en la persona. Sin embargo, seguramente no estará cerca un médico o enfermero cuando ocurra un paro cardiaco súbito fuera de un hospital o bien en los centros deportivos, por lo que otros profesionales de la salud, también deberían recibir esta formación.

Los resultados de la evaluación sobre el dominio en primeros auxilios que tenían profesores de una escuela primaria en Córdoba, España, fueron deficitarios, presentando conceptos erróneos sobre cómo curar heridas, la utilización del alcohol en la curación de heridas y desconocen el funcionamiento del desfibrilador externo automático. El promedio de respuestas acertadas obtenidas antes de la intervención educativa fue el 38,6 \%, y se incrementó después de la intervención al 76,2 \% (Alba-Martín, 2015).

En un estudio realizado en España a profesores de educación física sobre la percepción de formación profesional, todos los profesores manifestaron tener una percepción muy alta de sus competencias, en cuanto 
a la organización de la clase, el conocimiento del contenido de enseñanza, el conocimiento didáctico del contenido y la capacidad de liderazgo (Del Valle, De la Vega y Rodríguez, 2015). Sin embargo, con frecuencia la percepción de los profesores, difiere en gran medida con los resultados de evaluaciones formales de sus conocimientos y destrezas.

Para desarrollar un perfil profesional más integral en el campo de la salud, Chile y otros países de Latinoamérica consideran la RCP como un contenido obligatorio en los currícula de medicina. sin embargo, la RCP debería incluirse en todos los programas educativos relacionados con la salud.

Las personas que han recibido capacitación reciente o en los últimos cinco años, es más probable que brinden reanimación cardiopulmonar que aquellas que recibieron capacitación hace más de cinco años o bien que nunca han recibido capacitación. La falta de auxilio ante un paro cardiaco súbito puede presentarse principalmente por la falta de capacitación, falta de conocimiento sobre los signos de un paro cardiaco, un sentido de incompetencia y temor para proporcionar ayuda, temor en causar un daño a la persona y el temor a ser inculpado por la muerte de la persona (Medtronic Foundation, 2013).

La reanimación cardiopulmonar es una actividad psicomotora que puede ser aprendida y desarrollada, con el entrenamiento adecuado, puesto que demanda una técnica específica y una condición física adecuada, implicando fuerza y resistencia, con una demanda intensa de energía y activación general del cuerpo (Universidad de Arizona, 2014).

Por lo que surgieron las siguientes preguntas de investigación:

¿Está preparado el personal de los centros deportivos para brindar los primeros auxilios ante un ataque cardiaco súbito?

El estudio se plantea desde la perspectiva del campo laboral, esto es a partir de las personas que prestan servicios en centros deportivos, con o sin formación profesional y desde una proyección profesional, por medio del análisis curricular de los programas de estudio relacionados con el campo de la actividad física y el deporte.

\section{OBJETIVO}

Identificar la preparación del personal que labora en los gimnasios y centros deportivos para proporcionar reanimación cardiopulmonar ante un ataque cardiaco súbito.

El presente estudio aporta información indispensable para la generación de conocimiento en la línea "Muerte súbita en el deporte". La información recolectada contribuye al diagnóstico indispensable para emprender acciones remediales y preventivas para enfrentar el riesgo de muerte súbita en el deporte.

\section{MARCO TEÓRICO}

El paro cardiorespiratorio es la interrupción brusca e inesperada, de la respiración y de la actividad mecánica del corazón, que es potencialmente reversible (García, González, López y Santos, 2011). El paro cardiaco súbito (PCS) es "la principal causa de muerte entre deportistas jóvenes. En muchos casos, aunque la víctima tiene una apariencia saludable en el momento del colapso, sólo una de cada 10 sobrevive". El paro cardíaco súbito en personas jóvenes a menudo está causado por un problema cardíaco subyacente (Medtronic Foundation, 2013).

El paro cardiaco súbito se presenta por lo general por una afección cardiaca no diagnosticada, es súbito porque no se espera, sorprendiendo a la víctima en cualquier lugar, sin embargo, ante una emoción o esfuerzo físico intenso se incrementa la posibilidad de riesgo de muerte súbita. El paro cardiaco súbito pude padecerlo desde un niño hasta un anciano, aunque su frecuencia es mayor en los hombres, no distingue origen, ni raza. 
La reanimación cardiopulmonar $(\mathrm{RCP})$ o resucitación cardiopulmonar básica se compone de un conjunto de maniobras en secuencia cuya finalidad es identificar una situación de paro cardiaco respiratorio, activar el sistema de emergencias y sustituir la función respiratoria y sustituir mecánicamente el funcionamiento del sistema respiratorio y cardiovascular hasta la recuperación o el inicio de una RCP avanzada a través del uso de un desfibrilador automático (García, González, López y Santos, 2011). Se define como el uso terapéutico de la corriente eléctrica en la fibrilación ventricular o taquicardia ventricular sin pulso. La fibrilación ventricular es la causa más frecuente asociado al paro cardiaco en el adulto, por lo tanto, la desfibrilación debe considerarse parte del soporte vital básico en de la RCP (Nodal, López y De La Llera, 2006).

La aplicación oportuna de las maniobras de reanimación cardiopulmonar y la desfibrilación en personas con paro cardiaco han sido ampliamente demostradas como auxilio para la reanimación efectiva, permite mejorar el pronóstico de vida (García, González, López y Santos, 2011; Rojas, Aizman, Arab, Utili y Andresen, 2012). Se ha demostrado que la inmediatez del inicio de las maniobras de RCP, como la duración y calidad de estas maniobras son factores que determinan la supervivencia de la persona (García, González, López y Santos, 2011). Un elevado porcentaje de las maniobras de RCP, aproximadamente el 40 \%, se producen en la vía pública. Algunos de estos casos, pueden ser reanimados con éxito si la reanimación cardiopulmonar básica se inicia lo más pronto posible, entres el primer minuto y 4 minutos, y es seguida en el plazo de 10 minutos de una adecuada reanimación cardiopulmonar avanzada por personal sanitario (Fradejas y Pérez, 2013).

La reanimación cardiopulmonar avanzada "agrupa el conjunto de conocimientos, técnicas y maniobras dirigidas a proporcionar el tratamiento definitivo a las situaciones de PCR, optimizando la sustitución de las funciones respiratorias y circulatorias hasta el momento en que estas se recuperen" (Nodal, López y De La Llera, 2006, p.1).

...recomendaciones de International Liaison Committee on Resuscitation (ILCOR). Así, mediante los registros se evaluó: evaluación del nivel de conciencia, solicitud de ayuda a terceros, evaluación de la presencia signos de circulación (respiración espontánea y pulso arterial carotideo), postura del operador y sus brazos, amplitud y frecuencia del masaje cardiaco, ejecución ininterrumpida de éste, frecuencia y efectividad de la ventilación asistida, solicitud precoz y uso adecuado del desfibrilador, el tiempo trascurrido antes de la primera descarga y seguimiento de la secuencia de reanimación ABCD. En la evaluación de los signos de circulación se observó la apertura correcta de la vía aérea, la búsqueda de respiración espontanea (escuchar, sentir y ver la respiración) y del pulso arterial en las arterias carotideas. La solicitud de ayuda se consideró correcta cuando esta consideraba al menos la solicitud de un carro de paro o desfibrilador. Las compresiones torácicas fueron consideradas efectivas cuando: eran realizadas a una frecuencia de 100 a 120 compresiones por minuto ininterrumpidas y con una profundidad de 3,5 a $5 \mathrm{~cm}$. Las ventilaciones a su vez, se consideraron efectivas cuando se realizaban con un intervalo de 2 por cada 30 compresiones torácicas, si lograban elevar el tórax del maniquí y daban tiempo para la salida de aire (aproximadamente 2 segundos). La solicitud del desfibrilador debía ocurrir antes de 30 segundos de haber evaluado al paciente y descarga eléctrica antes de 30 segundos de haber evaluado el ritmo (FV) en el monitor. Se consideró correcto cuando la energía seleccionada antes de la descarga era de 360 jouls. (Rojas, Aizman, Arab, Utili y Andresen, 2012, p.74-75).

A continuación (Ver Tabla 2), se presenta una guía de reanimación cardiopulmonar para adultos y niños, quienes son las personas que frecuentan con mayor frecuencia los centros deportivos (Escalante, 2010). 


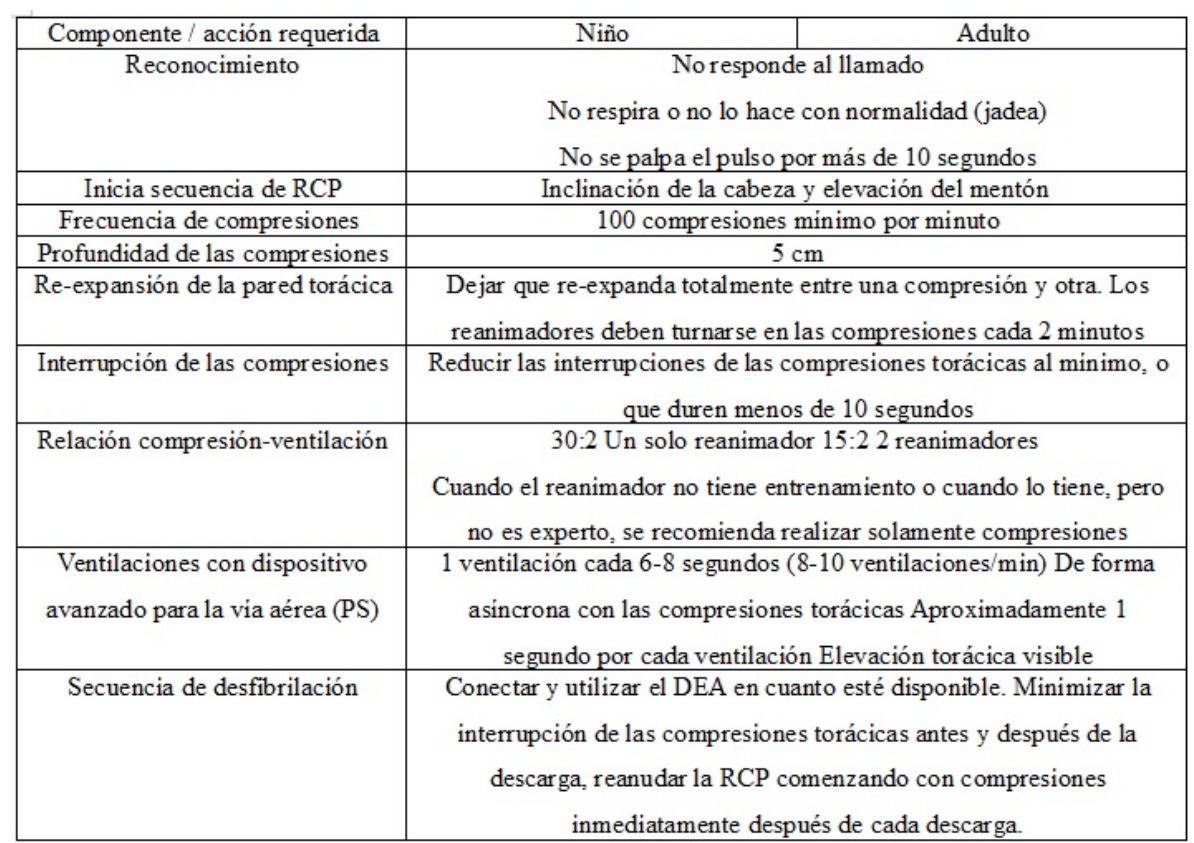

Tabla 2. Maniobras críticas de RCP básico para el personal de salud. Escalante (2010)

Las maniobras de RCP básica es una técnica fácil de aprender y realizar, siguiendo la secuencia de supervivencia. Los sistemas educativos deben conseguir que todos los profesionales que pueden ser posibles testigos directos de una parada cardiaca sepan realizar estas maniobras. Especialmente los más jóvenes, ya que "como muestran numerosos estudios desde los 13 años se pueden aprender estas técnicas, siendo quizás los jóvenes entre 15-16 años la población ideal para realizar esta formación” (Fradejas y Pérez, 2013, p.17).

\section{MÉTODO}

La presente investigación corresponde a un estudio descriptivo con enfoque mixto. La primera etapa desarrollada fue a través de métodos cualitativos, la cual consistió en el análisis curricular del plan de estudios de seis universidades que ofrecen carreras relacionadas con la actividad física y el deporte. La segunda etapa fue a través de la administración de una encuesta a centros deportivos y gimnasios, que recolectó datos cuantitativos y cualitativos.

La muestra fue aleatoria considerándose a los 20 centros deportivos y gimnasios más concurridos en el municipio de Hermosillo, Sonora, México. La muestra definitiva fue de 12, lo que representó un $60 \%$ de los centros deportivos y gimnasios. Es importante señalar que dos gimnasios se negaron a proporcionar la información solicitada por considerar confidencial los datos de sus usuarios y resguardar los intereses de sus propietarios.

Se elaboró una encuesta, que fue revisada y validada en consenso por la academia de Ciencias Psicopedagógicas del Deporte y de la Actividad Física del Departamento de Ciencias del Deporte y de la Actividad Física de la Universidad de Sonora. La encuesta se conformó por 15 reactivos, cuantificables y seis reactivos cualitativos con preguntas de desarrollo que solicitaban especificar y argumentar sus respuestas.

Las encuestas fueron aplicadas de manera directa visitando los gimnasios, centros deportivos e instituciones formadoras de profesionales en actividad física y deporte, algunas fueron enviadas por correo electrónico a los responsables de unidades académicas en las universidades participantes para su administración.

A continuación, se presenta la cantidad de estudiantes consultados y sus universidades (Ver Tabla, 3): 


\begin{tabular}{|c|c|}
\hline $\begin{array}{c}\text { Institución formadora de profesionales en el } \\
\text { campo de la actividad fisica y deporte }\end{array}$ & $\begin{array}{c}\text { Cantidad de estudiantes } \\
\text { consultados }\end{array}$ \\
\hline Universidad de Sonora & 100 \\
\hline Escuela Normal de Educación Fisica (Sonora) & 100 \\
\hline Universidad Estatal de Sonora & 100 \\
\hline Instituto Tecnológico de Sonora & 100 \\
\hline Total & 400 \\
\hline
\end{tabular}

Tabla 3. Cantidad de estudiantes consultados por institución. Elaboración propia

Se realizó un análisis curricular a 19 planes de estudio, relacionados al campo de la actividad física y el deporte, para identificar si la RCP está incluida como contenido obligatorio en algún programa educativo. Los planes educativos analizados fueron los siguientes:

a) Licenciatura en Educación Física (Escuela Normal de Educación Física)

b) Licenciatura en Entrenamiento Deportivo (Universidad Estatal de Sonora)

c) Licenciatura en Cultura Física y Deporte (Universidad de Sonora)

d) Licenciatura en Ciencias del Ejercicio Físico (Instituto Tecnológico de Sonora)

e) Licenciatura en Actividad Física y Deporte (Universidad Autónoma de Baja California)

f) Licenciatura en Educación Física y Deporte (Universidad de Colima)

g) Universidad Autónoma de Ciudad Juárez

h) Universidad Autónoma de Aguascalientes

i) Universidad Autónoma de Chihuahua

j) Universidad de Guadalajara

k) Centro Universitario de la Costa / Universidad de Guadalajara

1) Universidad Autónoma del Carmen

m) Benemérita Universidad Autónoma de Puebla

n) Universidad Veracruzana

o) Universidad de Morelos

p) Universidad Juárez del Estado de Durango

q) Universidad Autónoma de Nuevo León

r) Universidad Autónoma de Sinaloa (Licenciatura en Educación Física)

s) Universidad Autónoma de Sinaloa (Licenciatura en Entrenamiento Deportivo)

\section{RESULTADOS}

\section{Análisis de los centros deportivos y gimnasios}

Para resguardar el anonimato y confidencialidad de la información proporcionada por los responsables de los gimnasios y centros deportivos, los datos se presentan codificados con letras en orden alfabético (Ver Tabla 4). 


\begin{tabular}{|c|c|}
\hline Código & Promedio de usuarios por mes \\
\hline A & 1200 \\
\hline B & 300 \\
\hline C & 500 \\
\hline D & 500 \\
\hline E & 150 \\
\hline F & 200 \\
\hline G & 150 \\
\hline I & 150 \\
\hline J & 120 \\
\hline K & 150 \\
\hline L & 500 \\
\hline & 80 \\
\hline
\end{tabular}

Tabla 4. Código y promedio de usuarios por mes en los centros deportivos y gimnasios.

Elaboración propia

A continuación, se presentan los datos generales de los usuarios de los centros deportivos y gimnasios consultados (edad mínima, edad máxima, promedio de edad, promedio de lesiones leves al mes y promedio de lesiones graves al mes).

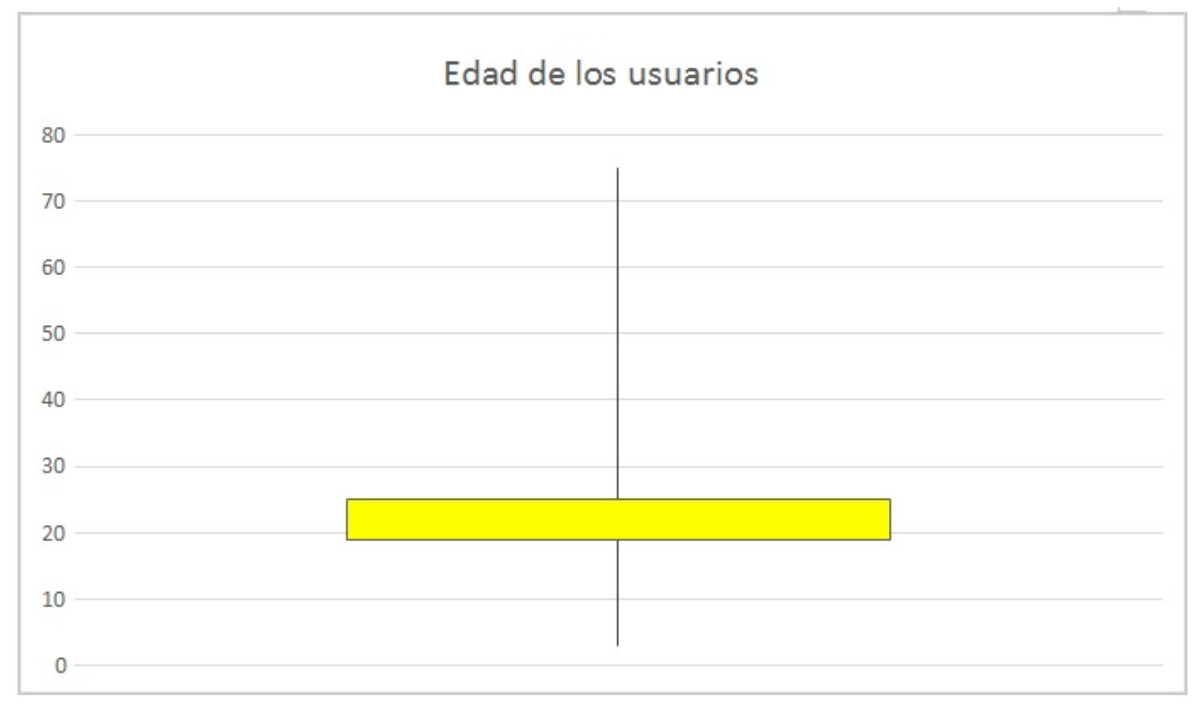

Figura 1. Edad de los usuarios

Elaboración propia

Considerando todas las repuestas obtenidas por los responsables de los centros deportivos y gimnasios, la edad mínima registrada fue de tres años y una edad máxima de 75 años; la mediana y moda de la edad promedio de los usuarios fue 25 años; el promedio de lesiones leves al mes fue de 2,25. Las personas encuestadas reportaron que no se ha presentado lesiones graves en los centros deportivos o gimnasios. Sin embargo, expresaron que las lesiones más frecuentes que se han presentado son contracturas y desgarres musculares, torcedura de tobillo, lumbalgias y escoriaciones. Se observa que en todos los centros deportivos o gimnasios se atiende una población adulta, en los casos de A y B, se reportó una edad máxima de usuarios de 75 años. 


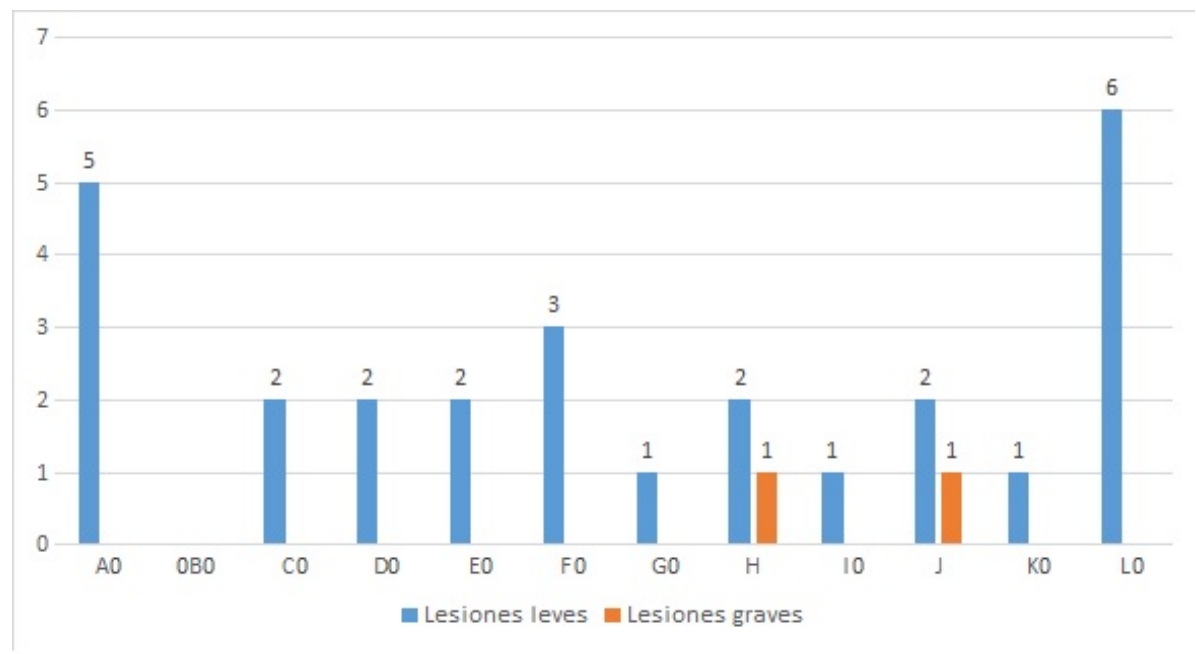

Figura 2. Lesiones de los usuarios por centro deportivo Elaboración propia

Como se observa en la Figura 2, solamente en dos gimnasios se reportó un accidente grave. Los responsables de los centros deportivos y gimnasios aseguraron que nunca se ha presentado un ataque cardiaco súbito dentro de las instalaciones.

Sobre la capacitación del personal del centro deportivo o gimnasio referente al dominio de las maniobras de RCP y conocimiento del uso del desfibrilador automático externo se encontró que en dos centros ningún trabajador tiene conocimientos al respecto (Ver Figura 2).

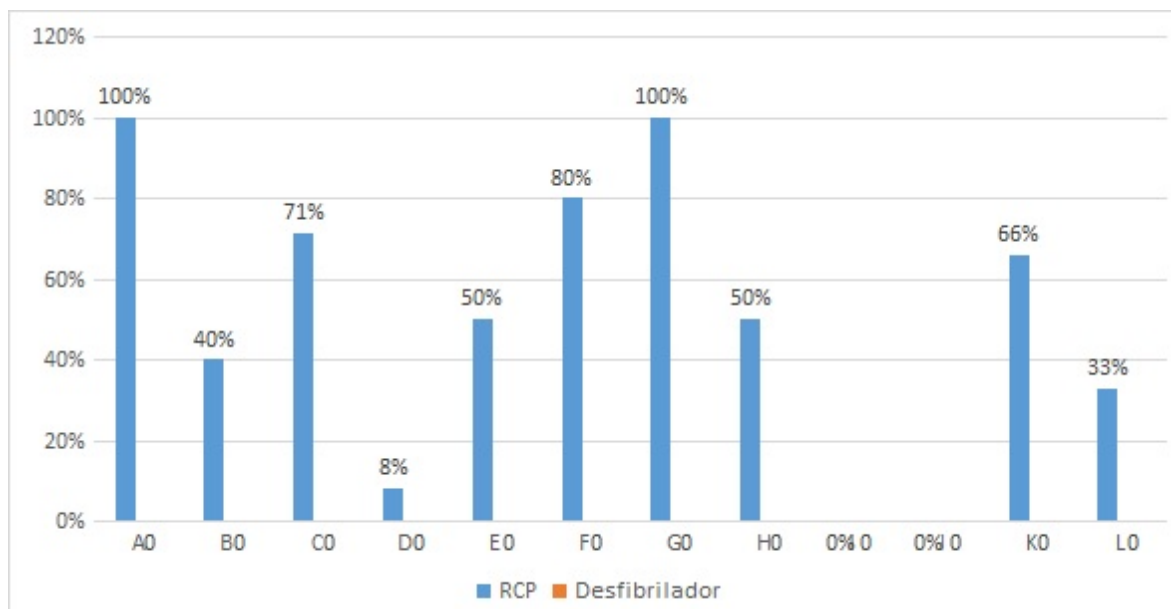

Figura 3. Porcentaje del personal del centro deportivo que ha recibido capacitación sobre las maniobras de RCP y el uso del desfibrilador externo automático por centro deportivo. Elaboración propia

Los centros deportivos encuestados presentaron una población máxima de 20 empleados y una mínima de tres; el promedio de edad máximo de los empleados fue de 50 años y una edad promedio mínimo de 20; siete de los doce centros deportivos confirmaron haber recibido capacitación en reanimación cardiopulmonar en los últimos tres años, lo que representa un 58,33\%, dos de los centros deportivos confirmaron que el $100 \%$ de su personal conoce como realizar reanimación cardiopulmonar (A, G); sin embargo, el total de los centros deportivos confirmó que su personal desconoce la manera de operar un desfibrilador automático externo y que no cuentan en las instalaciones con el equipo. 
La mayoría de los responsables del centro deportivo o gimnasio explicaron que no cuentan con un desfibrilador externo automático, por su alto costo.

El total de los encuestados aseguró que le gustaría que el personal recibiera capacitación sobre el procedimiento de reanimación cardiopulmonar y el uso del desfibrilador externo automático.

\section{Análisis de los planes de estudio}

Únicamente los planes de estudio de la carrera de Entrenamiento Deportivo de la Universidad Autónoma de Sinaloa, la Universidad Veracruzana, y el Centro Universitario de la Costa / Universidad de Guadalajara, contemplan como obligatoria la asignatura de Primeros Auxilios, donde se incluye el tema de RCP.

El plan de estudios de la Licenciatura en Actividad Física y Deporte de la Universidad de Colima incluye la asignatura de Primeros Auxilios, es una asignatura Optativa del Bloque de materias, Bases Biológicas de la Motricidad Humana, aunque si incluye contenidos específicos sobre RCP, no todos los estudiantes la cursan por ser optativa. Los contenidos de la asignatura Primeros Auxilios son los siguientes (Universidad de Colima, 2015):
a) Seguridad personal y Evaluación del lesionado
b) Signos Vitales
c) Obstrucción de la vía aérea
d) Respiración de salvamento
e) Reanimación Cardiopulmonar
f) Heridas y hemorragias
g) Esguinces, luxaciones, fracturas y vendajes
h) Urgencias ambientales y médicas comunes

\section{DISCUSIÓN}

Ofrecer servicios recreativos, de entrenamiento de fuerza y acondicionamiento físico, demanda una gran responsabilidad y preparación del centro deportivo o gimnasio y de todo el personal que trabaja, no sólo de los instructores.

El hecho de que dos empresas que se dedican a ofrecer servicios de entrenamiento de fuerza (gimnasio) oculten información y no hayan accedido a responder la encuesta, aun explicando el anonimato y uso académico de la información es injustificado, puesto que la transparencia de la seguridad que brindan debe comunicarse abiertamente a cualquier usuario o persona interesada. Afortunadamente, no se reportan lesiones graves, ni ataque cardiaco súbito en ningún centro deportivo o gimnasio.

Es importante señalar que los riesgos siempre están presentes en un centro deportivo; sin embargo, estos se incrementan en una población adulta mayor en aumento, por lo que se deben tomar medidas precautorias y preventivas en todo momento, aún más cuando se atiende a personas de la tercera edad. Los centros deportivos o gimnasios se atiende una población adulta correspondientes a la segunda adultez y en los casos de A y B, existen usuarios de hasta 75 años de edad.

Aunque el estudio, no pretendió evaluar el nivel de destreza en las maniobras de primeros auxilios, específicamente en la RCP, sólo consultar si habían recibido capacitación, se observa que no todo el personal ha sido capacitado y que las instituciones formadoras de profesionales en actividad física y deporte, no están egresando a sus estudiantes con esta destreza de primeros auxilios, situación que se identifica como una debilidad que se sugiere atender. Con base en las evaluaciones del dominio de las maniobras en RCP a personal de salud especializado (Almeida, Araújo, Dalri y Araujo, 2011; Rojas, Aizman, Arab, Utili y Andresen, 2012) y docentes (Alba-Martín, 2015) los cuales obtuvieron un promedio reprobatorio, resulta 
conveniente que todo el personal reciba nuevamente la capacitación en RCP, puesto que no es una destreza que practiquen con frecuencia, permitiendo de esta manera adquirir un mejor entrenamiento. Se infiere que los responsables de los centros deportivos y gimnasios encuestados con respecto a la capacitación en RCP, contestaron en su mayoría, en conformidad con las expectativas de los investigadores del presente estudio, puesto que sólo dos personas proporcionaron detalles de la formación recibida y de la organización que proporcionó la capacitación. Se confirma que la percepción de los responsables de los centros deportivos tiende a ser muy alta en relación a los conocimiento y competencias de los instructores y personal de trabajo, tal como lo señalaron en su investigación Del Valle, De la Vega y Rodríguez (2015).

La capacitación en RCP y en el uso de un desfibrilador automático externo, así como su adquisición, resulta urgente, la prevención debe ser parte de las políticas operativas de los centros deportivos y gimnasios; para no justificar estas acciones después de una tragedia.

\section{CONCLUSIÓN}

De manera contundente se confirma que el personal de los centros deportivos y gimnasios no está preparado para brindar los primeros auxilios a una persona que es víctima de un ataque cardiaco súbito. De igual manera, los futuros profesionales del campo de la actividad física y el deporte, muy pocos conocen como realizar las maniobras de RCP, y en los planes de estudio, consideran estos contenidos como asignaturas optativas, por lo que no todos los egresados han recibido capacitación al respecto.

El tiempo de respuesta que se tiene en un gimnasio o centro deportivo, para recibir reanimación cardiopulmonar ente un ataque cardiaco súbito fuera de un hospital, está supeditado al proporcionado por los servicios de emergencia (Cruz Roja), con un promedio de arribo de 15 minutos, por lo que la víctima no tiene posibilidades de sobrevivir, puesto que se suma el tiempo de haberse presentado el paro cardiaco y el tiempo en que se tardaron en realizar la llamada al servicio de emergencia.

Se identificó que sólo dos de cada 100 estudiantes de carreras relacionadas con educación física, ejercicio físico, entrenamiento deportivo y cultura física, confirman haber recibido capacitación en la RCP.

Sin embargo, los responsables de los centros deportivos confirman que cuentan con personal capacitado en RCP, pero desconocen el uso del desfibrilador automático externo. Además, se confirma que en ningún centro deportivo se cuenta con desfibrilador automático externo para brindar auxilio en caso de paro cardiaco súbito.

El $100 \%$ de los responsables de los centros deportivos confirmaron estar interesados en recibir capacitación para realizar maniobras de la RCP y el uso de desfibriladores.

La gran responsabilidad que implica ser un profesional de la salud, específicamente en el campo de la actividad física y el deporte, por la prescripción del ejercicio físico que se realiza como parte de las tareas básicas, demanda una sólida preparación para trabajar con todos los grupos etarios, en condición de salud o con ausencia de ella. La precaria o deficiente preparación en primeros auxilios, en el caso específico de la RCP, representa una debilidad identificada en el perfil de egreso y profesional, puesto que las emergencias si bien no son con mucha frecuencia, los casos que se presenten de paro cardiaco súbito, podrían tener mayor posibilidad de supervivencia si se recibe de manera inmediata el auxilio adecuado.

\section{Recomendaciones}

Se sugiere que el personal que labora en los centros deportivos y gimnasios reciba capacitación sobre las maniobras de reanimación cardiopulmonar y el uso del desfibrilador. Se propone que el $100 \%$ del personal adquiera las destrezas para ejecutar las maniobras de RCP, con base en los parámetros y procedimientos internacionales. 
Se sugiere que en todos los centros deportivos y gimnasios se cuente con carteles que instruyan en el proceso de la RCP y tengan de manera accesible un desfibrilador automático externo.

Lamentablemente en las carreras relacionadas con actividad física y deporte en el noroeste de México, no se incluye la RCP como un contenido curricular obligatorio, situación que limita su intervención ante un paro cardiaco súbito. A pesar de ello, los profesionales de la salud y educadores físicos, podrían compensar esta limitación en su perfil profesional, atendiendo los contenidos de RCP y el uso del desfibrilador externo automático en programas no formales.

\section{REFERENCIAS}

Alba-Martín, R. (2015). Educación para la salud en primeros auxilios dirigida al personal docente del ámbito escolar. Enfermería universitaria, 12(2), 88-92. Recuperado de https://dx.doi.org/10.1016/j.reu.2015.04.004

Almeida, A., Araújo, I., Dalri, M. y Araujo, S. (2011). Conocimiento teórico de los enfermeros sobre parada cardiorrespiratoria y resucitación cardiopulmonar en unidades no hospitalarias de atención de urgencia y emergencia. Revista Latinoamericana Enfermagen 19(2). Recuperado de http://www.scielo.br/pdf/rlae/v19n2 /es_06.pdf

Arimany-Manso, J. y Bayés de Luna, A. (2011). Muerte súbita en el deporte. Revista Española de Medicina Legal, 37(4), 131-133. Recuperado de http://goo.gl/bsxMNS

Boraita, A. (2002). Muerte súbita y deporte. ¿Hay alguna manera de prevenirla en los deportistas? Revista Española de Cardiología, 55(4). Recuperado de http://www.revespcardiol.org/es/sudden-death-and-sport-is/articulo/13 029693/

Del Valle, S., De la Vega, R. y Rodríguez, M. (2015). Percepción de las competencias profesionales del docente de educación física en primaria y secundaria. Revista Internacional de Medicina y Ciencias de la Actividad Física y el Deporte, 15(59), 507-526. Recuperado de http://cdeporte.rediris.es/revista/revista59/artpercepcion593.htm

Escalante, R. (2010). Guías de reanimación cardiopulmonar. Acta Médica Peruana, 27(4). Recuperado de http://ww w.scielo.org.pe/pdf/amp/v27n4/a12v27n4

Fradejas, V. y Pérez, P. (2013). Importancia de una comunidad educativa formada en técnicas de Reanimación Cardiopulmonar, Nuberos Cientifica, 3(10), 13-17. Fundación de Enfermería de Cantabria. Recuperado dehttp://www.enfermeriacantabria.com/enfermeriacantabria/web/articulos/10/77

García, M., González, R., López, J. y Santos, A. (2011). La reanimación cardiopulmonar y la atención inicial a las urgencias y emergencias pediátricas. Revista Pediatría atención Primaria, 13(20). Recuperado de http://scielo.i sciii.es/scielo.php?script $=$ sci_arttext\&pid $=$ S1 139-76322011000400022

Hoyos, G., Castro, P., Hoyos, L. Chávez, M. y Plasencia, L. (2011). Influencia de la actividad física en los niveles de triglicéridos y colesterol en niños con sobrepeso y obesidad. Revista Ciencia, Deporte y Cultura Física, 3, 16-25. Universidad de Colima-Universidad Iberoamericana.

Martín-Recio, F. (2009). Prevención y primeros auxilios en educación física. Innovación y Experiencias Educativas, 21. Recuperado de http://www.csi-csif.es/andalucia/modules/mod_ense/revista/pdf/Numero_21/FRANCIS CO\%20JESUS_MARTIN_RECIO01.pdf

Martínez-Morilla, J.A., Ruiz-Caballero, J.A., Brito-Ojeda, E. y Navarro-Valdivielso, M.E. (2012). Producción científica sobre cardiología y deporte: ranking de revistas (2010) y excelencia. Revista Internacional de Medicina y Ciencias de la Actividad Física y el Deporte, 12(46), 299-312. Recuperado de http://cdeporte.rediris.es/revist a/revista46/artproduccion284.htm

Medtronic Foundation. (2013). Every Second Counts. Every Action Matters, A Community Response Planning Guide for Sudden Cardiac Arrest. HeartRescue Project. Recuperado de http://www.heartrescueproject.com/ wcm/groups/mdtcom_sg/@mdt/@corp/@fndn/@heartrescue/documents/images/hrp-brochure.pdf

Nodal, P., López, J. y De La Llera, G. (2006). Paro cardiorrespiratorio (PCR). Etiología. Diagnóstico. Tratamiento. Revista Cubana de Cirugia, 45, 3-4. Recuperado de http://scielo.sld.cu/pdf/cir/v45n3-4/cir19306.pdf 
Rojas, L., Aizman, A., Arab, J., Utili, F. y Andresen, M. (2012). Reanimación cardiopulmonar básica: conocimiento teórico, desempeño práctico y efectividad de las maniobras en médicos generales. Revista Médica de Chile, 140(1), 73-77. Recuperado de http://www.scielo.cl/pdf/rmc/v140n1/art10.pdf

Universidad de Arizona. (2014). Be a lifesaver. Sarver Hearth Center. Recuperado de http://www.azdhs.gov/documents/preparedness/emergency-medical-services-trauma-system/save-hearts-a z-registry-education/newsletter/2014/cpr-pocket-spanish.pdf

Universidad de Colima (2015). Documento curricular. Licenciado en Educación física y Deporte. Facultad de Ciencias de la Educación. México: Universidad de Colima.

Recepción: 27 Diciembre 2017

Aprobación: 21 Enero 2018

La aceptación de colaboraciones por parte de la revista implica la cesión no exclusiva de los derechos patrimoniales de los autores a favor del editor, quien permite la reutilización, luego de su edición (postprint), bajo Licencia Creative Commons Atribución-NoComercial-CompartirIgual 4.0 Internacional (https:// creativecommons.org/licenses/by-nc-sa/4.0/).

CC BY-SA 